\title{
Seuil d'application de la méthode du Gradex
}

\section{Extrapolating a flood frequency curve using the "Gradex" method}

\author{
C. Michel \\ G. Oberlin
}

CEMAGREF, Antony

Une présentation générale de la méthode du Gradex (Guillot, Duband, 1967) largement utilisée en France souligne les difficultés rencontrées dans l'utilisation pratique de la méthode. Une de ces difficultés est le choix de la fréquence à partir de laquelle la méthode est applicable. Il semble difficile de résoudre ce problème sans introduire un nouveau modèle. Le but de la présente communication est de proposer un tel modèle permettant un usage plus fiable de la méthode du Gradex.

A general presentation of the Gradex method (Guillot, Duband, 1967) widely used in France underlines the difficulties encountered in the practical use of the method. One of these difficulties is the frequency choicie upon which the method applies. It is often difficult to resolve this problem without having to introduce a new model. The purpose of this article is to propose a model which will enable the Gradex method to be used more reliably.

\section{Introduction}

Actuellement, il existe un besoin toujours plus grand d'une meilleure connaissance des risques, même très faibles. On est donc conduit à déterminer des débits qui ont une très faible probabilité de dépassement annuelle $\left(10^{-4}, 10^{-6} \ldots\right)$. Or, les distributions statistiques établies à partir des observations ne peuvent pas être considérées comme valides bien au-delà de la durée totale des observations. Un moyen d'extrapolation plus raisonné est donc nécessaire. La méthode du gradex fournit un tel moyen. Comme toute méthode d'extrapolation, celle-ci souffre de diverses incompréhensions et aussi de difficultés d'application, dont certaines ont été rappelées récemment (Beran, 1981).

Dans cette note, on s'intéresse au problème posé par la non-connaissance du point de départ adéquat pour l'extrapolation proprement dite par le gradex des pluies. C'est ce point de départ que nous appelons le seuil, en fréquence, d'application de la méthode.

\section{Rappel sur le gradex}

La méthode est bien connue en France grâce aux nombreux efforts de publication de ses auteurs (Guillot et Duband, 1967, 1973). On en rappelle ci-après les principales caractéristiques. On suppose que pour un bassin versant on dispose d'une durée caractéristique de crue, et l'on raisonnera dans tout ce qui suit sur des volumes de pluies et de débit se rapportant à cette durée : nous désignons par $P$ la pluie maximale de l'année et par $Q$ le débit maximal de la même année. Notons au passage que les réalisations de $P$ et $Q$ n'appartiennent pas forcément au même événement averse-crue.

La méthode du gradex repose sur les hypothèses suivantes :

$$
\begin{gathered}
Q=P-J \text { si } \quad J<P \\
Q=0 \text { si } J \geqslant P
\end{gathered}
$$


$J$ est une variable aléatoire asymptotiquement indépendante de $P$

$P$ suit une loi de Gumbel :

$$
F_{P}=\exp (-\exp (-(P-b) / a))
$$

$a$ est appelé le gradex de la distribution (diminutif de gradient exponentiel).

Dans ces conditions, on va montrer que $Q$ suit asymptotiquement une loi de Gumbel de même gradex $a$ (CEMAGREF, 1972).

Soit $F_{J}$ la fonction de distribution $J$. Pour $q>0$, la fonction de distribution de $Q, F_{Q}$, est donnée par :

$$
\begin{aligned}
& F_{Q}(q)=\operatorname{Prob}\{Q \leqslant q\}= \\
& \qquad \int_{0}^{\infty}\left(d\left(F_{J}(J)\right) \int_{-\infty}^{q+J} d\left(F_{P}(P)\right)\right. \\
& F_{Q}(q)=\int_{0}^{\infty} e^{-e^{-\frac{q+J-b}{a}} d F_{J}(J)}
\end{aligned}
$$

En notant $J(F)$ la fonction inverse de $F_{\mathcal{N}}(J)$ on a :

$$
\begin{gathered}
\mathrm{F}_{\mathrm{Q}}(q)=\int_{0}^{1} e^{-e^{-\frac{q+J(F)-b}{a}} d F} \\
F_{Q}(q)=\int_{0}^{1} e^{-e^{-\frac{q-b}{a}}} \frac{J(F)}{a} d F \\
F_{Q}(q)=\int_{0}^{1}\left(e^{-e^{-\frac{q-b}{a}}}\right)^{e^{-\frac{J(F)}{a}}} d F
\end{gathered}
$$

Or, si $q$ est assez grand, $e^{-e^{-\frac{q-b}{a}}}$ est très peu inférieur à 1. Posons cette quantité égale à $1-\varepsilon$ :

$$
\begin{aligned}
& \mathrm{F}_{\mathrm{Q}}(q)=\int_{0}^{1}(1-\varepsilon)^{e^{-\frac{J(F)}{a}}} d F \\
& \cong \int_{0}^{1}\left(1-\varepsilon e^{-e^{-\frac{J(F)}{a}}} d F\right.
\end{aligned}
$$

donc

$$
\mathrm{F}_{\mathrm{Q}}(q) \cong 1-\varepsilon \int_{0}^{1} e^{-\frac{J(F)}{a}} d F
$$

Posons :

$$
\begin{gathered}
\int_{0}^{1} e^{-\frac{J(F)}{a}} d F=c \\
F_{Q}(q) \cong 1-c \varepsilon \cong(1-\varepsilon)^{c}
\end{gathered}
$$

c'est-à-dire, en revenant à l'expression de $\varepsilon$ :

$$
\begin{gathered}
F_{Q}(q) \cong\left(e^{-e^{-\frac{q-b}{a}}}\right) \\
F_{Q}(q) \cong e^{-c e^{-\frac{q-b}{a}}} \\
F_{Q}(q) \cong e^{-e^{-\frac{q-b-a \log c}{a}}}
\end{gathered}
$$

On voit donc qu'asymptotiquement la distribution de $Q$ est bien une loi de Gumbel de même gradex $a$.

\section{Seuil d'application : la solution simplifiée}

Dans la pratique, on n'essaie pas de déterminer $J(F)$ du fait essentiellement de la difficulté qu'il y a à identifier les réalisations de $J$. Cette difficulté étant liée à la troncature de $J(J \leqslant P)$. Par conséquent, on suppose qu'au-delà d'une certaine valeur $q^{*}$, de fréquence au non-dépassement $F^{*}$, la distribution de $Q$ est sensiblement une loi de Gumbel de gradex $a$;

On a donc, pour $F>F^{*}$ :

$$
q=q^{*}-a \ln \left(\ln F / \ln F^{*}\right)
$$

En général $q^{*}$ et $F^{*}$ sont déterminés par la limite de validité de la distribution statistique de la variable $Q$. Si la variable $Q$ est valable jusqu'aux environs de

$$
F^{*}=-\frac{1}{2 n}
$$

On extrapole alors la distribution de $Q$ pour $F>F^{*}$ par la relation précédente (méthode du gradex).

\section{Critique de la solution simplifiée}

Dans de nombreux cas la distribution expérimentale des débits présente au point $\left(q^{*}, F^{*}\right)$ une " pente " beaucoup plus faible que le gradex $a$, lorsqu'on la représente sur un graphique de Gumbel comme ci-après :

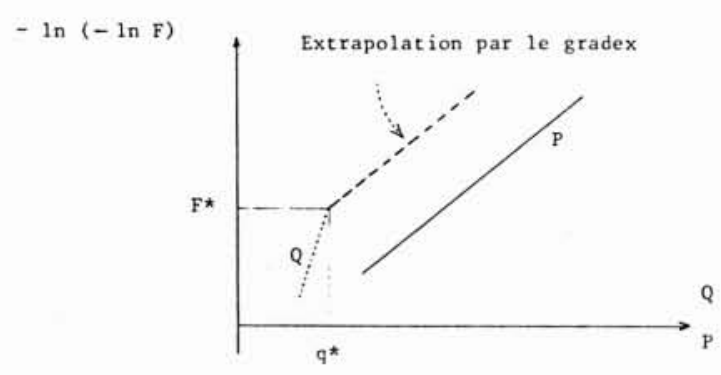

Dans ces conditions, l'application de la méthode conduit à une cassure irréaliste qui surestime visiblement les débits pour une fréquence donnée. Ce problème peut avoir deux causes :

i) la méthode ne s'applique pas à ce type de situation;

ii) le point $\left(q^{*}, F^{*}\right)$ est trop en-dessous du seuil à partir duquel l'extrapolation par le gradex est valide.

Dans ce deuxième cas, on peut dire que la méthode n'est valable que pour $F>F^{* *}$ avec $F^{* *}>F^{*}$. On présente ci-après une tentative pour résoudre ce problème. 


\section{Essai d'un modèle multiplicatif intermédiaire}

On a rappelé précédemment que le modèle $Q=P-J$ était délicat de vérification du fait de la non indépendance évidente de $P$ et de $J$ pour $P$ faible. Un modèle multiplicatif du type :

$$
Q=C^{\prime} \cdot P \quad \text { avec } \quad 0 \leqslant C^{\prime} \leqslant 1
$$

donne au moins à la variable $C^{\prime}$ un domaine de définition indépendant de $P$. Une transformation de variable sur $C^{\prime}$ semble utile et l'on peut écrire, par exemple :

$$
Q=\frac{P}{1+e^{c}} \text { avec } \quad-\infty<C<+\infty
$$

Un essai sur le bassin représentatif de l'Orgeval a montré que $C$ et $P$ pouvaient être considérés comme indépendants, au moins dans le domaine des fréquences observables et qu'une loi normale pouvait être ajustée à l'échantillon de $C$ (Colin et al., 1980). Essayons de déterminer la loi asymptotique de $Q$ dans le cas où $P$ suit une loi de Gumbel et $C$ une loi normale.

Notons $F_{c}(C)$ la fonction de distribution de la variable $C$. On a pour expression de la fonction de distribution de $Q$ :

On en déduit donc :

$$
F_{Q}(Q)=\int_{-\infty}^{+\infty} d F_{c} \int_{-\infty}^{Q(1+c c)} d F_{p}
$$

$$
F_{Q}(Q)=\int_{-\infty}^{+\infty} F_{P}\left(Q\left(1+e^{c}\right)\right) d F_{c}(C)
$$

soit, comme $F_{P}(x)=\exp (-\exp (-((x-b) / a)))$ :

$$
\begin{aligned}
& F_{Q}(Q)=\int_{-\infty}^{\infty} \exp (-\exp \\
&(-((Q(1+\exp C)-b) / a))) d F_{c}(C)
\end{aligned}
$$

Utilisons la fonction inverse de la distribution $C$ : $C=C(F)$, soit :

$$
\begin{aligned}
F_{Q}(Q)=\int_{0}^{1} \exp (-\exp \\
(-((Q(1+\exp C(F))-b) / a))) d F
\end{aligned}
$$

La quantité sous le signe somme peut s'écrire :

$$
\left(F_{P}(Q)\right)^{\exp (-Q \exp C(A / a)}
$$

Or, si $Q$ est grand, $F_{P}(Q)$ est proche de 1 .

Comme on l'a vu précédemment, avec un nombre $\alpha$ proche de 1 , on a :

$$
\int_{0}^{1} \alpha^{f(x)} d x \cong \alpha^{\int_{0}^{1} f(x) d x}
$$

et donc :

$$
F_{Q}(Q) \cong\left(F_{P}(Q)\right) \int_{0}^{1} \exp (-Q \exp C(F) / a) d F
$$

On peut utiliser à nouveau la même propriété pour la quantité sous le signe somme :

$$
\int_{0}^{1} \exp (-Q \exp C(F) / a) d F=\int_{0}^{1}\left(e^{-Q / a}\right)^{\exp C(F)} d F
$$

et par conséquent :

$$
\begin{array}{r}
\int_{0}^{1} \exp (-Q \exp C(F) / a) d F \cong e^{-\frac{\mathrm{Q}}{a} \int_{0}^{1} \exp C(F) d F} \\
e \int_{0}^{1} \exp C(F) d F=\int_{-\infty}^{+\infty} \exp C d F_{c}(C)
\end{array}
$$

Or, $F_{c}(C)$ est une loi de Gauss dont nous noterons les paramètres $m$ et $\mathrm{s}$.

Par conséquent :

$$
d F_{c}(C)=\frac{1}{\sqrt{2 \pi} s} \exp \left(-\frac{1}{2}\left(\frac{C-m}{s}\right)^{2}\right) d C
$$

donc

$$
\begin{aligned}
& \int_{0}^{1} \exp C(F) d F \\
& =\int_{-\infty}^{+\infty} \exp C \cdot \frac{1}{\sqrt{2 \pi} s} \exp \left(-\frac{1}{2}\left(\frac{C-m}{s}\right)^{2}\right) d C \\
& =\frac{1}{\sqrt{2 \pi} s} \int_{-\infty}^{+\infty} \exp \left(-\frac{1}{2}\left(\frac{C-m}{s}\right)^{2}+C\right) d C \\
& =\frac{\exp \left(m+\frac{s^{2}}{2}\right)}{\sqrt{2 \pi} s} \int_{-\infty}^{+\infty} \exp ( \\
& \left.-\frac{1}{2}\left(\frac{C-m-s^{2}}{s}\right)^{2}\right) d C \\
& =\exp \left(m+\frac{s^{2}}{2}\right)
\end{aligned}
$$

en revenant à la distribution de $Q$, on obtient :

$$
F_{Q}(Q) \cong F_{P}(Q)^{e^{-\frac{Q}{a} \exp \left(m+\frac{s 2}{2}\right)}}
$$

ce qui donne, après quelques calculs :

$$
F_{Q}(Q) \cong F_{P}\left[Q\left(1+\exp \left(m+\frac{s^{2}}{2}\right)\right)\right]
$$

Autrement dit, la loi de $Q$ est asymptotiquement une loi de Gumbel mais cette fois-ci de gradex :

$$
a /\left(1+\exp \left(m+\frac{s^{2}}{2}\right)\right)
$$

Le modèle $\mathrm{Q}=\frac{\mathrm{P}}{1+e^{c}}$ conduit bien à une loi de Gumbel pour $Q$, mais de gradex différent de celui des pluies. D'autre part, l'hypothèse de l'indépendance entre $C$ et $P$ pour les très fortes valeurs de $P$ peut paraitre suspecte. En conséquence, il ne faut pas utiliser ce modèle multiplicatif vers les fréquences très rares.

On peut cependant, et c'est sa destination première et son objectif, l'utiliser comme étape intermédiaire entre l'ajustement statistique direct sur l'échantillon de $Q$ et l'extrapolation par le gradex. On notera que le résultat simple auquel nous sommes arrivés n'est pas utilisable à cette fin d'extrapolation intermédiaire et antérieure à celle du gradex et que l'on devra recourir au calcul numérique 
de la distribution de $Q$ à partir de l'expression générale donnée au départ (2). On dispose donc ici d'une méthode permettant d'améliorer le seuil d'application du gradex en allant au-delà de la méthode simplifiée (seuil à $q^{*}$ et $F^{*}$ ); mais elle ne donne pas directement le point recherché $\left(q^{* *} F^{* *}\right)$ qu'il faut encore déterminer arbitrairement. Le progrès est cependant sensible.

\section{Autre solution proposée}

Devant les relatives difficultés rencontrées pour résoudre encore imparfaitement ce problème de la détermination du point $\left(q^{* *}, F^{* *}\right)$ par la méthode précédente du modèle multiplicatif, on peut recourir à la recette simple ci-après. On sait qu'une relation de rendement très utilisée, en particulier par le Soil Conservation Service des Etats-Unis, s'écrit :

$$
Q=\frac{(P-c)^{2}}{P+d}
$$

Avec $c+d>0$ de façon que $Q$ soit toujours défini pour $P>c$. On peut admettre que cette relation reste asymptotiquement valable pour les quantiles de ces variables et que l'on a, pour $P_{F}>c$ :

$$
Q_{F} \cong \frac{\left(P_{F}-c\right)^{2}}{P_{F}+d}
$$

L'intérêt de cette formulation est d'exprimer que $Q$ suit asymptotiquement une loi de Gumbel de même gradex que $P$.

Le développement de la division de $\left(P_{F}-c\right)^{2}$ par $\left(P_{F}+d\right)$ donne, en effet :

$$
Q_{F}=P_{F}-(2 c+d)+\frac{(c+d)^{2}}{P_{F}}-\frac{(c+d)^{2} d}{P_{F}^{2}}+\ldots
$$

On voit bien que $P_{F}$ et $Q_{F}$ ne diffèrent asymptotiquement que d'une constante.

L'extrapolation utilisée consiste à caler $c$ et $d$ de façon à éviter la fâcheuse cassure au point $\left(q^{*}, F^{*}\right)$.

Supposons que l'on ait ajusté une loi de Gumbel sur l'échantillon disponible de la variable $Q$ et soit $a_{Q}$ le gradex de cette distribution (gradex des débits observés). Notons $u$ la variable de Gumbel $u=-\ln (-\ln F)$.

$$
q^{*}=\frac{\left(P^{*}-c\right)^{2}}{P^{*}+d}
$$

et, d'autre part, $\quad\left(\frac{d Q_{F}}{d u}\right)=a_{q}$

avec $a_{Q}<a$

$$
\frac{d Q_{F}}{d u}=\frac{d P_{F}}{d u} \cdot\left[1-\left(\frac{c+d}{P_{F}+d}\right)^{2}\right]
$$

avec $\frac{d P_{F}}{d u}=a$ soit :

$$
a_{Q}=a\left[1-\left(\frac{c+d}{P^{*}+d}\right)^{2}\right]
$$

Les relations (3) et (4) vont permettre de déterminer $c$ et $d$, ce qui explicitera l'extrapolation cherchée.

Posons :

$$
\sqrt{1-\frac{a_{Q}}{a}}=\alpha \quad 0<\alpha<1
$$

On en déduit :

$$
\frac{c+d}{P^{*}+d}=\alpha
$$

$(c+d$ devant toujours être positif).

Soit :

$$
d=\frac{\alpha P^{*}-c}{1-\alpha}
$$

d'où, en reportant dans (3) :

$$
q^{*}=\frac{\left(P^{*}-c\right)^{2}}{P^{*}+\frac{\alpha P^{*}-c}{1-\alpha}}
$$

c'est-à-dire : $q^{*}=(1-\alpha)\left(P^{*}-c\right)$

d'où :

$$
c=P^{*}-\frac{q^{*}}{1-\alpha}
$$

et

$$
d=\frac{q^{*}}{(1-\alpha)^{2}}-P^{*}
$$

Ce qui donne finalement l'expression recherchée pour l'extrapolation :

$$
Q_{F}=\frac{\left[P_{F}-P^{*}+\frac{q^{*}}{1-\alpha}\right]^{2}}{P_{F}-P^{*}+\frac{q^{*}}{(1-\alpha)^{2}}}
$$

Cette expression est donc en principe valable pour $F>F^{*}$ et permet d'extrapoler la distribution observée des $Q$ au-delà du couple $\left(F^{*}, q^{*}\right)$, tout en intégrant la méthode du gradex proprement dite puisqu'elle y conduit asymptotiquement.

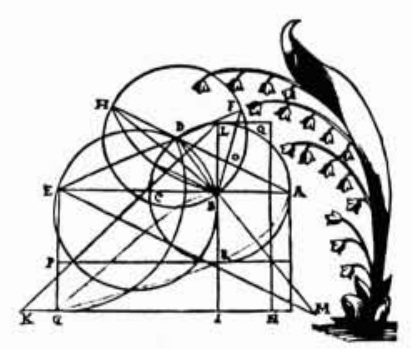




\section{Conclusion}

La méthode du gradex est une méthode très simple et très rustique et c'est pourquoi on continue à la préférer à d'autres méthodes plus en vogue dans les pays anglosaxons. Dans les améliorations qu'on peut tenter de lui apporter quant à son application pratique et en particulier pour préciser son seuil d'application, il faut veiller à ne pas nuire à cette qualité de simplicité.

C'est la raison pour laquelle cette note propose deux solutions d'amélioration de la détermination du seuil d'extrapolation par le gradex : la première, dite modèle multiplicatif, est relativement longue à mettre en œuvre et ne résoud pas complètement le problème; mais elle est la plus satisfaisante car elle est physiquement et statistiquement cohérente et exploite au mieux les informations disponibles (observations). La seconde est simple à mettre en œuvre et résoud complètement le problème en assurant une totale continuité entre la distribution statistique observée des débits et le gradex proprement dit; elle repose cependant sur des hypothèses un peu simples et n'exploite pas toute l'information disponible.

\section{Références}

Guillot P. et Duband D. (1967). - La méthode du gradex pour le calcul de la probabilité des crues à partir des pluies Floods and their computations (Leningrad Symposium, 1967), pp. 560-569: AISH Publ. $n^{\circ} 84$.

Guillot P. (1973). - Précisions sur la méthode du gradex Utilisation de l'information hydrométéorologique pour l'évaluation de la crue de projet. $X I^{*}$ Congres international des grands barrages, Madrid, 11-15 juin 1973, Q. 41 R 8, pp. 123144 .

Beran M. (1981). - The "gradex" method, letter to the Editor, IAHS Bulletin 26, 2, 6/1981, pp. $223-224$.

C.T.G.R.E.F. (CEMAGREF) (1972). - L'application de la méthode du gradex à l'estimation des crues de faible fréquence, Antony.

Colin E. et Michel C. (1980). - Approche statistique du coefficient d'écoulement et utilisation pour la prédétermination des crues. La Houille Blanche, $\mathrm{n}^{\circ} 4 / 5$, 1980, pp. 251-258. 